\title{
Productivity of fodder beet (Beta vulgaris var.Crassa) cultivars affected by nitrogen and plant spacing
}

\author{
Muna E.Khogali ${ }^{1}$, Yassin M.I.Dagash ${ }^{2}$ and Mahgoub G.EL-Hag ${ }^{3}$ \\ ${ }^{1}$ Department of Science and Technology of Animal Production, Faculty of Science and \\ Technology of Animal Production, Sudan University of Science and Technology, \\ www.sustech.edu Khartoum North (Kuku), Sudan'Email:muna_eltayeb@yahoo.com. Tel: \\ +249 122321022 \\ ${ }^{2}$ Department of Field Crops Sciences, Faculty of Agricultural Studies, Sudan University of \\ Science and Technology, www.sustech.edu Shambat, Sudan. Email: dagash50@yahoo.com. \\ Tel: +249 913354525 \\ ${ }^{3}$ Department of Animal Production, Faculty of Agriculture,Al zaiem Alazhary University,
}

\begin{abstract}
A field experiment was conducted in the (2007/08 - 2008/09) seasons to study the effects of nitrogen fertilization and plant spacing on yields and its components of three fodder beet cultivars. The experiment was a randomized complete block design (RCBD) in split-split plots arrangement. Nitrogen treatments at a rate of $0,40,80$ and $120 \mathrm{~kg} \mathrm{~N} / \mathrm{ha}$ were assigned to the main plots applied eleven days from planting. Plant spacing (15, 20 and $25 \mathrm{~cm}$ between holes) and fodder beet cultivars (Voroshenger, Anisa and Polyproductiva) were allotted to the sub and sub-sub-plots, respectively. Nitrogen application increased root fresh weight; dry weights of shoot, root; green and dry fodder yields significantly $(p<0.05)$ in the first season. Nitrogen had no significant effects on shoot fresh weight. Wider spacing significantly $(p<0.05)$ increased root dry weight in the first season. Green and dry fodder yields were significantly $(p<0.05)$ increased under closer spacing in the second season. Spacing had no significant effects on root fresh weight, shoot fresh weight, and shoot dry weight. Cultivars exhibited highly significant $(p<0.01)$ differences in shoot dry weight in both seasons. Cultivars affected shoot fresh weight significantly $(p<0.05)$ in the first season and highly significantly $(p<0.01)$ in the second one. Fresh and dry weight of root, green and dry fodder yields were not significantly affected by cultivars. Shoot dry weight was significantly greater under higher nitrogen levels and wider spacing $\left(\mathrm{N}_{2} \mathrm{~S}_{2}\right.$ and $\left.\mathrm{N}_{3} \mathrm{~S}_{3}\right)$ in the second season. Nitrogen and cultivars interaction resulted in significant $(p<0.05)$ increase in root dry weight attained by Voroshenger and Polyproductiva cultivars under $80 \mathrm{~kg} / \mathrm{N} / \mathrm{ha}\left(\mathrm{N}_{2} \mathrm{~V}_{1}\right.$ and $\left.\mathrm{N}_{2} \mathrm{~V}_{3}\right)$ in the first and second season, respectively. Polyproductiva sown at $25 \mathrm{~cm}$ apart $\left(\mathrm{S}_{3} \mathrm{~V}_{3}\right)$ significantly $(\mathrm{p}<0.05)$ attained the greatest root dry weight in the second season.
\end{abstract} \begin{abstract}
INTRODUCTION
Fodder beet (Beta vulgaris var.Crassa), a member of the chenopodiaceae family, is also known as mangel (Martin, 1976). The fodder beet is a native of Mediterranean area and was grown as a root crop in Germany and Italy as early as the Sixteenth Century. The crop was introduced into the United States in colonial days (Kipps, 1970). It is World-wide in temperate zones up to $55^{\circ} \mathrm{N}$ (WWW.biolaie.unihambura.de, 2006).
\end{abstract}

Key words: Nitrogen, Spacing, Cultivars, Fodder beet, Yield
The production of forage crops is very important for livestock production in the Sudan, which contributes largely to the national income. Animal production in the Sudan depends mainly on natural range which is affected by rain fluctuations and low quality grasses. This necessitates the introduction of irrigated forage crops in the irrigated schemes and in farms around cities like Khartoum.

There are many constrains facing forage production in the Sudan, like lack of information of forage cultivars and technological packages. Suggested 
solutions for these problems are application of technological packages, integration of animal production with forage production and introduction of new forage species of high yield (Khair, 1999) especially during periods of forage shortage like late winter and early summer.

Fodder beet offers a higher yield potential than any other arable fodder crop (Anonymous, 2006) and when grown under suitable conditions can produce almost 20 t/ha ${ }^{-1}$ dry matter yield (DAF, 1998) and also yields more than $80 \mathrm{t} / \mathrm{ha}$ and this makes it popular in many countries like New Zealand, Germany, America, Australia, Syria and Egypt (Shalaby, et al., 1989). It contains $10-15 \%$ dry matter and may yield 20 t/ha of dry matter in one harvest as compared to 13-15 t/ha from four cuts of grass (Kiely et al. 1991).

Nitrogen is often the most limiting factor in crop production. Hence, application of fertilizer nitrogen results in higher biomass yield (Blumenthal et al., 2008).

The above and below growth parts (leaves and roots) are used to feed the animals but, the main fodder is tuberous roots (Ibrahim, 2005). Therefore the optimum population which produces maximum leaves and roots yield must be carefully determined.

Fodder beet is a good forage especially during the critical period of forage shortage such as early summer season in the Sudan. The objectives of this study are to study the effects of nitrogen fertilizer and spacing on yield and yield components of fodder beet to provide information on cultural practices concerning this important crop under Sudan conditions.

\section{MATERIALS AND METHODS}

A field experiment was conducted for two consecutive winter seasons of November (2007-2008 and 2008-2009), in the Demonstration Farm of the College of Agricultural Studies, Sudan University of Science and Technology at Shambat, Khartoum North (latitude $15^{\circ} 40^{\prime} \mathrm{N}$ and Longitude $32^{\circ} 32^{\prime}$ E).The soil of the experimental site is clay loam, characterized by low nitrogen content $(0.05)$ and $\mathrm{Ph}$ of 7.61-8.26. A three factors experiment was conducted in a randomized complete block design (RCBD) in split-split plots arrangement and four replications. The main plots were allotted to nitrogen fertilization and the sub-plots to the spacing. Three fodder beet cultivars were used: Voroshenger (Hungarian), Anisa (Italian) and Polyproductiva (Polish). The land was disc- ploughed, harrowed twice, leveled and ridged $70 \mathrm{~cm}$ apart. The size of the plot was $3 \times 3 \mathrm{~m}$, consisting of four ridges of $3.21 \mathrm{~m}$ length. The seeds were sown manually on the shoulder of the ridge at a rate of $4.6 \mathrm{~kg} / \mathrm{ha}$ (three seeds per hole) on November 30 and 13 in the first and second season, respectively.

The crop was irrigated at 7-10 days intervals depending on the temperature, relative humidity and soil moisture conditions. Nitrogen fertilization in the form of urea $(46 \% \mathrm{~N})$, was broadcast once before the third irrigation ( 11 days from planting) at a rate of 0 (N0) as control, 40 (N1), 80 (N2), and 120 (N3) kg $\mathrm{N} /$ ha. Triple superphosphate $\left(46 \% \mathrm{P}_{2} \mathrm{O}_{5}\right)$ was added preplanting at a rate of $100 \mathrm{~kg} / \mathrm{fed}$. as agronomic practice. Three spacing between holes: $15 \mathrm{~cm}$ (S1), $20 \mathrm{~cm}$ (S2) and $25 \mathrm{~cm}$ (S3) were used. Hand thinning to one plant per hole and resowing by the removed seedlings were done simultaneously after 5-6 weeks from planting during both seasons. Manual weeding by a hand implement "Nagama" was done, after 5 weeks from planting in the two seasons.

At harvest (3 - 3.5 months from sowing), when plants showed signs of maturity which is indicated by leaf yellowing and partial drying of the lower leaves, three plants per plot from the inner two ridges were randomly hand-pulled to determine fresh and dry weights $(\mathrm{g})$ of shoot and root. The green fodder yield was obtained by weighing plants in $1.4 \mathrm{~m}^{2}$ and then transformed to ton per hectare. To obtain the dry fodder yield, the weighed plants in $1.4 \mathrm{~m}^{2}$ were dried in an oven at $105^{\circ} \mathrm{C}$ for $24 \mathrm{hrs}$, and then they were converted to ton per hectare.

Analysis of variance (ANOVA) appropriate for the split-split plot design was applied (Gomez and Gomez, 1984). The treatment means were compared using Duncan Multiple Range Test (D.M.R.T) and Least Significant Difference (LSD) procedures at 5\% level using MSTAT Computer Program v.4 (1986).

\section{RESULTS AND DISCUSSION:}

Shoot fresh weight: Table (I) illustrates the effects of nitrogen, spacing and cultivars on shoot fresh weight. Shoot fresh weight was insignificantly greater under higher nitrogen level $(120 \mathrm{Kg} \mathrm{N} / \mathrm{ha}$ ) and wider spacing $(25 \mathrm{~cm})$. However, both Voroshanger and Polyproductiva cultivars obtained significantly greater shoot fresh weights than Anisa in the first season and highly significantly greater shoot weight in the second season. This agrees with the work of Abdalla (1999) on two Sugar beet cultivars, who reported that 
cultivar Pamela resulted in greater foliage fresh weight than cultivar Sonja.

Root fresh weight: Root fresh weight was significantly and positively affected by nitrogen application and the greatest weight was recorded for N3 $(120 \mathrm{Kg} \mathrm{N} / \mathrm{ha})$ as shown in table I. This finding is in agreement with that obtained by Mokadem (2000) who reported that supplying beet plants with nitrogen fertilizer up to $100 \mathrm{~kg} \mathrm{~N} / \mathrm{fed}$. significantly increased root fresh weight/plant. Similar trend was observed by Ibrahim et al. (2005); El-Shafai (2000) and Ismail (2002).Spacing had no significant effect on root fresh weight (Table I). Despite that, wider spacing of $25 \mathrm{~cm}$ $\left(\mathrm{S}_{3}\right)$ recorded the highest root fresh weight during the second season. Cultivars were not significantly different in root fresh weight which agrees with the findings reported by Abdalla (2008) and Nadaf et al. (1998a and 1998b).

Shoot dry weight: Raising nitrogen fertilization levels from 40, 80 to $120 \mathrm{~kg} \mathrm{~N} / \mathrm{ha}$ has resulted in a significantly higher shoot dry weight (Table 1). This increase could be due to the increase in the amount of metabolites synthesized by plants due to the effect of nitrogen in enhancing photosynthesis and hence dry matter accumulation. This leads to more growth and consequently accumulation of more photosynthesis assimilates. Similar observations were reported by El-Shafai (2000); Ismail (2002); Nemeat Alla et al. (2002) and Nafei (2004) .Plants grown at different spacing were not significantly different regarding shoot dry weight (Table 1). But, the productivity of the individual plant was enhanced under lower density planting and under dense planting the single plant produced much less shoot dry weight.

In both seasons cultivars showed highly significant differences in average shoot dry weight. Higher weight was recorded for Voroshenger and Polyproductiva than Anisa cultivar (Table 1). This is confirmed by Abdalla (1999) working on two sugar beet cultivars. The interaction effect between nitrogen fertilization and spacing on shoot dry weight (Table 2) showed the superiorityof $25 \mathrm{~cm}\left(\mathrm{~S}_{3}\right)$ between holes under $120 \mathrm{Kg} \mathrm{N} / \mathrm{ha}\left(\mathrm{N}_{3}\right)$ and spacing of $20 \mathrm{~cm}\left(\mathrm{~S}_{2}\right)$ under $80 \mathrm{Kg} \mathrm{N} /$ haTherefore the significant highest shoot dry weight/plant was achieved by sowing at spacing of $25 \mathrm{~cm}\left(\mathrm{~S}_{3}\right)$ with nitrogen fertilization level of $120 \mathrm{Kg} \mathrm{N} / \mathrm{ha}\left(\mathrm{N}_{3}\right)$ in the first season of $25 \mathrm{~cm}\left(\mathrm{~S}_{3}\right)$ between holes under $120 \mathrm{Kg} \mathrm{N} / \mathrm{ha}\left(\mathrm{N}_{3}\right)$ and spacing of $20 \mathrm{~cm}\left(\mathrm{~S}_{2}\right)$ under $80 \mathrm{Kg} \mathrm{N} / \mathrm{ha}$.Therefore, the significant highest shoot dry weight/plant was achieved by sowing at spacing of $25 \mathrm{~cm}\left(\mathrm{~S}_{3}\right)$ with nitrogen fertilization level of $120 \mathrm{Kg} \mathrm{N} / \mathrm{ha}\left(\mathrm{N}_{3}\right)$ in the first season.

Root dry weight: Root dry weight was significantly increased as nitrogen rate increased. The greatest weight was obtained by adding $120 \mathrm{Kg} \mathrm{N} / \mathrm{ha}$ (N3) over the control (N0) and $40 \mathrm{Kg}$

$\mathrm{N} / \mathrm{ha}$ (N1) in the first season (Table 1). This increase could be attributed to the role of nitrogen in enhancing plant growth (Ouda, 2005) and consequently accumulation of more assimilates (Ibrahim et al., 2005).Root dry weight was increased significantly when sown at wider spacing of $25 \mathrm{~cm}$ (Table 1). This agrees with Hassanin and Ramadan (1999) who stated that $30 \mathrm{~cm}$ hill spacing increased root weight $(\mathrm{g})$ over $20 \mathrm{~cm}$ within rows. No significant differences were observed between the cultivars regarding root dry weight (Table 1$)$. This result is in line with those obtained by Mustafa (2007) and Abdallah (2008) in the Sudan. Root dry weight was significantly affected by interactive effect between nitrogen and cultivars during the second season, when polyproductiva fertilized with $80 \mathrm{~kg} \mathrm{~N} / \mathrm{ha}\left(\mathrm{N}_{2}\right.$ $\mathrm{CV}_{3}$ ) attained the greatest root dry weight (Table III). This may be attributed to the cultivar difference in efficiency of nitrogen utilization. Spacing $X$ cultivars interaction effect on root dry weight was significant in the second season when Polyproductiva cultivar $\left(\mathrm{CV}_{3}\right)$ planted at $25 \mathrm{~cm}$ a part had the greatest root dry weight than other combination of cultivars and spacing (Table 4). Polyproductiva attained the greatest shoot fresh weight which increased photosynthesis besides the more nutrients in the wider spacing and hence the greatest root dry weight.

Green fodder yield: Increasing nitrogen level up to $120 \mathrm{~kg} / \mathrm{ha}\left(\mathrm{N}_{3}\right)$ significantly increased green yield over the control by $29.9 \%$ in the first season (Table 1).This result was supported by Ibrahim (1998) and Basha (1999) who concluded that application of nitrogen significantly increased top and root yields/fed.Also, Mahmoud et al. (1990) found that top and root yield were increased due to increasing nitrogen levels up to $80 \mathrm{~kg} \mathrm{~N} / \mathrm{fed}$. and Nemeat Alla et al. (2002) showed the same trend. Increasing 
Agric. Biol. J. N. Am., 2011, 2(5): 791-798

Table 1: Effects of nitrogen fertilization, spacing, and cultivars on yield and yield components of fodder beet for 2007-2008 and 2008-2009

\begin{tabular}{|c|c|c|c|c|c|c|c|c|c|c|c|c|c|}
\hline \multirow{2}{*}{\multicolumn{2}{|c|}{ Treatments }} & \multicolumn{2}{|c|}{$\begin{array}{l}\text { Shoot fresh weight } \\
(\mathrm{g})\end{array}$} & \multicolumn{2}{|c|}{$\begin{array}{l}\text { Root fresh weight } \\
\text { (g) }\end{array}$} & \multicolumn{2}{|c|}{$\begin{array}{l}\text { Shoot dry weight } \\
\text { (g) }\end{array}$} & \multicolumn{2}{|c|}{$\begin{array}{c}\text { Root dry weight } \\
\text { (g) }\end{array}$} & \multicolumn{2}{|c|}{$\begin{array}{l}\text { Green fodder yield } \\
\text { (ton/ha) }\end{array}$} & \multicolumn{2}{|c|}{ Dry fodder yield (ton/ha) } \\
\hline & & $2007 / 08$ & $2008 / 09$ & $2007 / 08$ & $2008 / 09$ & $2007 / 08$ & $2008 / 09$ & $2007 / 08$ & $2008 / 09$ & $2007 / 08$ & $2008 / 09$ & $2007 / 08$ & $2008 / 09$ \\
\hline Nitrogen & $\begin{array}{l}\text { N0 } \\
\text { N1 } \\
\text { N2 } \\
\text { N3 }\end{array}$ & $\begin{array}{l}476.65 \mathrm{a} \\
558.61 \mathrm{a} \\
699.10 \mathrm{a} \\
650.95 \mathrm{a}\end{array}$ & $\begin{array}{l}489.03 \mathrm{a} \\
509.58 \mathrm{a} \\
701.63 \mathrm{a} \\
719.86 \mathrm{a}\end{array}$ & $\begin{array}{c}800.23 \mathrm{~b} \\
971.36 \mathrm{ab} \\
1030.66 \mathrm{ab} \\
1152.09 \mathrm{a}\end{array}$ & $\begin{array}{l}1031.35 \mathrm{a} \\
1131.53 \mathrm{a} \\
1419.73 \mathrm{a} \\
1360.54 \mathrm{a}\end{array}$ & $\begin{array}{c}46.44 \mathrm{~b} \\
50.86 \mathrm{ab} \\
58.53 \mathrm{a} \\
58.11 \mathrm{a}\end{array}$ & $\begin{array}{l}48.17 \mathrm{a} \\
46.67 \mathrm{a} \\
65.30 \mathrm{a} \\
66.26 \mathrm{a}\end{array}$ & $\begin{array}{c}114.22 \mathrm{~b} \\
128.65 \mathrm{~b} \\
131.82 \mathrm{ab} \\
149.96 \mathrm{a}\end{array}$ & $\begin{array}{l}128.77 \mathrm{a} \\
154.89 \mathrm{a} \\
161.87 \mathrm{a} \\
154.73 \mathrm{a}\end{array}$ & $\begin{array}{r}106.82 \mathrm{~b} \\
129.11 \mathrm{ab} \\
144.74 \mathrm{ab} \\
152.40 \mathrm{a}\end{array}$ & $\begin{array}{c}129.70 \mathrm{a} \\
141.31 \mathrm{a} \\
180.85 \mathrm{a} \\
175.30 \mathrm{a}\end{array}$ & $\begin{array}{c}13.56 \mathrm{c} \\
15.26 \mathrm{bc} \\
16.07 \mathrm{ab} \\
17.79 \mathrm{a}\end{array}$ & $\begin{array}{l}15.02 \mathrm{a} \\
17.22 \mathrm{a} \\
19.41 \mathrm{a} \\
18.63 \mathrm{a}\end{array}$ \\
\hline $\begin{array}{l}\text {-test } \\
\mathrm{SE} \pm\end{array}$ & & $\begin{array}{c}N S \\
70.11\end{array}$ & $\begin{array}{c}\text { NS } \\
75.24\end{array}$ & $\begin{array}{c}* \\
75.27\end{array}$ & $\begin{array}{c}\text { NS } \\
117.83\end{array}$ & $\begin{array}{c}* \\
3.39 \\
\end{array}$ & $\begin{array}{c}\text { NS } \\
7.04 \\
\end{array}$ & $\begin{array}{c}* \\
5.91\end{array}$ & $\begin{array}{l}\text { NS } \\
11.85\end{array}$ & $11.63^{*}$ & $\begin{array}{c}\text { NS } \\
15.55\end{array}$ & $\begin{array}{c}* \\
0.72\end{array}$ & $\begin{array}{c}\mathrm{NS} \\
1.40 \\
\end{array}$ \\
\hline Spacing & $\begin{array}{l}\text { S1 } \\
\text { S2 } \\
\text { S3 }\end{array}$ & $\begin{array}{l}511.18 \mathrm{a} \\
580.36 \mathrm{a} \\
697.47 \mathrm{a}\end{array}$ & $\begin{array}{l}583.28 \mathrm{a} \\
592.11 \mathrm{a} \\
639.69 \mathrm{a}\end{array}$ & $\begin{array}{c}874.13 \mathrm{a} \\
964.72 \mathrm{a} \\
1126.92 \mathrm{a}\end{array}$ & $\begin{array}{l}1164.35 \mathrm{a} \\
1206.04 \mathrm{a} \\
1336.96 \mathrm{a}\end{array}$ & $\begin{array}{l}48.69 \mathrm{a} \\
51.12 \mathrm{a} \\
60.65 \mathrm{a}\end{array}$ & $\begin{array}{c}54.00 \mathrm{a} \\
57.37 \text { as } \\
58.42 \mathrm{a}\end{array}$ & $\begin{array}{l}121.75 \mathrm{~b} \\
125.18 \mathrm{~b} \\
146.55 \mathrm{a}\end{array}$ & $\begin{array}{l}140.14 \mathrm{a} \\
148.85 \mathrm{a} \\
161.22 \mathrm{a}\end{array}$ & $\begin{array}{l}138.53 \mathrm{a} \\
130.95 \mathrm{a} \\
130.31 \mathrm{a}\end{array}$ & $\begin{array}{c}174.76 \mathrm{a} \\
154.13 \mathrm{ab} \\
141.49 \mathrm{~b}\end{array}$ & $\begin{array}{l}17.10 \mathrm{a} \\
15.11 \mathrm{a} \\
14.80 \mathrm{a}\end{array}$ & $\begin{array}{c}19.41 \mathrm{a} \\
17.61 \mathrm{ab} \\
15.69 \mathrm{~b}\end{array}$ \\
\hline $\begin{array}{l}\text { F-test } \\
\text { SE } \pm\end{array}$ & & $\begin{array}{c}\text { NS } \\
66.20\end{array}$ & $\begin{array}{c}\text { NS } \\
31.99\end{array}$ & $\begin{array}{c}\text { NS } \\
81.35\end{array}$ & $\begin{array}{c}\text { NS } \\
77.00\end{array}$ & $\begin{array}{c}\text { NS } \\
4.21\end{array}$ & $\begin{array}{c}\text { NS } \\
2.95\end{array}$ & * 6.57 & $\begin{array}{l}\text { NS } \\
7.49\end{array}$ & $\begin{array}{c}\text { NS } \\
11.41\end{array}$ & $\stackrel{*}{*}$ & $\begin{array}{l}\mathrm{NS} \\
0.84\end{array}$ & ${ }^{*}$ \\
\hline Cultivars & $\begin{array}{l}\text { CV1 } \\
\text { CV2 } \\
\text { CV3 }\end{array}$ & $\begin{array}{l}644.20 \mathrm{a} \\
495.45 \mathrm{~b} \\
649.33 \mathrm{a}\end{array}$ & $\begin{array}{l}653.61 \mathrm{a} \\
502.26 \mathrm{~b} \\
659.21 \mathrm{a}\end{array}$ & $\begin{array}{c}976.22 \mathrm{a} \\
1003.58 \mathrm{a} \\
985.96 \mathrm{a}\end{array}$ & $\begin{array}{l}1199.90 \mathrm{a} \\
1251.89 \mathrm{a} \\
1255.56 \mathrm{a}\end{array}$ & $\begin{array}{l}57.10 \mathrm{a} \\
46.53 \mathrm{~b} \\
56.83 \mathrm{a}\end{array}$ & $\begin{array}{l}61.62 \mathrm{a} \\
47.51 \mathrm{~b} \\
60.67 \mathrm{a}\end{array}$ & $\begin{array}{l}131.47 \mathrm{a} \\
130.92 \mathrm{a} \\
131.10 \mathrm{a}\end{array}$ & $\begin{array}{l}147.28 \mathrm{a} \\
149.87 \mathrm{a} \\
149.87 \mathrm{a}\end{array}$ & $\begin{array}{c}135.29 \mathrm{a} \\
127.34 \mathrm{a} \\
137.16 \mathrm{a}\end{array}$ & $\begin{array}{l}157.03 \mathrm{a} \\
150.78 \mathrm{a} \\
162.56 \mathrm{a}\end{array}$ & $\begin{array}{l}15.98 \mathrm{a} \\
15.12 \mathrm{a} \\
15.92 \mathrm{a}\end{array}$ & $\begin{array}{l}17.71 \mathrm{a} \\
16.90 \mathrm{a} \\
18.11 \mathrm{a}\end{array}$ \\
\hline $\begin{array}{l}\text { F-test } \\
\text { SE } \pm\end{array}$ & & 31.97 & 27.19 & 49.96 & 51.48 & 2.41 & 2.47 & 5.01 & $\begin{array}{l}\text { NS } \\
5.17\end{array}$ & NS & NS & $\begin{array}{l}\text { NS } \\
0.53\end{array}$ & NS \\
\hline
\end{tabular}

*: Significant (5\%) $\quad$ **: Highly Significant (1\%)

Means within each column followed by the same letters are not significantly different at (5\%) level according to DMRT.

Nitrogen fertilization: N0 (Control) N1 $\quad$ N0 kg, N/ha) N2 (80 kg, N/ha) N3 (120 kg, N/ha)

Spacing: S1 $(15 \mathrm{~cm}) \quad \mathrm{S} 2(20 \mathrm{~cm}) \quad \mathrm{S} 3(25 \mathrm{~cm})$

Cultivars: CV1 (Voroshenger) CV2 (Anisa) CV3 (Polyproductiva)

Table 2: Effects of nitrogen $x$ spacing interactions on yield and yield components of fodder beet for 2007-08 and 2008-09

\begin{tabular}{|c|c|c|c|c|c|c|c|c|c|c|c|c|c|}
\hline \multicolumn{2}{|c|}{ Treatments } & \multicolumn{2}{|c|}{$\begin{array}{l}\text { Shoot fresh weight } \\
\text { (g) }\end{array}$} & \multicolumn{2}{|c|}{$\begin{array}{l}\text { Root fresh weight } \\
\text { (g) }\end{array}$} & \multicolumn{2}{|c|}{$\begin{array}{c}\text { Shoot dry weight } \\
\text { (g) }\end{array}$} & \multicolumn{2}{|c|}{$\begin{array}{c}\text { Root dry weight } \\
\text { (g) }\end{array}$} & \multicolumn{2}{|c|}{$\begin{array}{l}\text { Green fodder } \\
\text { yield(ton/ha) }\end{array}$} & \multicolumn{2}{|c|}{$\begin{array}{l}\text { Dry fodder yield } \\
\text { (ton/ha) }\end{array}$} \\
\hline & & $2007 / 08$ & $2008 / 09$ & $2007 / 08$ & $2008 / 09$ & $2007 / 08$ & $2008 / 09$ & $2007 / 08$ & $2008 / 09$ & $2007 / 08$ & $2008 / 09$ & $2007 / 08$ & $2008 / 09$ \\
\hline \multirow{3}{*}{ No } & S1 & $362.13 \mathrm{a}$ & $454.44 \mathrm{a}$ & $586.00 \mathrm{a}$ & $952.36 \mathrm{a}$ & $39.07 \mathrm{a}$ & $46.28 \mathrm{bc}$ & $94.10 \mathrm{a}$ & $116.78 \mathrm{a}$ & $94.82 \mathrm{a}$ & $140.68 \mathrm{a}$ & $13.33 \mathrm{a}$ & $16.31 \mathrm{a}$ \\
\hline & $\mathrm{S} 2$ & $490.98 \mathrm{a}$ & $460.28 \mathrm{a}$ & $887.97 a$ & $1074.17 \mathrm{a}$ & $46.65 \mathrm{a}$ & $44.30 \mathrm{bc}$ & $123.82 \mathrm{a}$ & $129.76 \mathrm{a}$ & $118.20 \mathrm{a}$ & $131.53 \mathrm{a}$ & $14.61 \mathrm{a}$ & $14.92 \mathrm{a}$ \\
\hline & S3 & $576.83 a$ & $552.36 \mathrm{a}$ & $926.71 \mathrm{a}$ & $1076.51 \mathrm{a}$ & $53.60 \mathrm{a}$ & $53.91 \mathrm{bc}$ & $124.75 \mathrm{a}$ & $139.76 \mathrm{a}$ & $107.39 \mathrm{a}$ & $116.90 \mathrm{a}$ & $12.74 \mathrm{a}$ & $13.83 \mathrm{a}$ \\
\hline \multirow{3}{*}{$\mathrm{N} 1$} & S1 & $468.97 \mathrm{a}$ & $562.22 \mathrm{a}$ & $855.45 \mathrm{a}$ & $1192.92 \mathrm{a}$ & $44.59 \mathrm{a}$ & $52.22 \mathrm{bc}$ & $119.63 \mathrm{a}$ & $153.06 \mathrm{a}$ & $132.44 \mathrm{a}$ & $175.52 \mathrm{a}$ & $16.42 \mathrm{a}$ & $20.53 a$ \\
\hline & $\mathrm{S} 2$ & $534.16 \mathrm{a}$ & $447.08 \mathrm{a}$ & $981.92 \mathrm{a}$ & $1101.53 \mathrm{a}$ & $50.68 \mathrm{a}$ & $44.79 \mathrm{bc}$ & $133.29 \mathrm{a}$ & $156.69 \mathrm{a}$ & $129.95 \mathrm{a}$ & $132.74 \mathrm{a}$ & $15.77 \mathrm{a}$ & $17.00 \mathrm{a}$ \\
\hline & S3 & $672.72 \mathrm{a}$ & $519.44 a$ & $1076.73 \mathrm{a}$ & $1100.14 \mathrm{a}$ & $57.30 \mathrm{a}$ & $43.00 \mathrm{c}$ & $133.02 \mathrm{a}$ & $154.93 \mathrm{a}$ & $124.95 \mathrm{a}$ & $115.68 \mathrm{a}$ & $13.60 \mathrm{a}$ & $14.14 \mathrm{a}$ \\
\hline \multirow{3}{*}{ N2 } & S1 & $568.75 a$ & $683.66 \mathrm{a}$ & $860.62 \mathrm{a}$ & $1324.54 \mathrm{a}$ & $53.45 \mathrm{a}$ & $60.36 \mathrm{abc}$ & $116.05 \mathrm{a}$ & $157.66 \mathrm{a}$ & $142.94 \mathrm{a}$ & $200.82 \mathrm{a}$ & $17.17 \mathrm{a}$ & $21.80 \mathrm{a}$ \\
\hline & $\mathrm{S} 2$ & $673.05 a$ & $788.44 \mathrm{a}$ & $915.28 \mathrm{a}$ & $1354.72 \mathrm{a}$ & $52.23 \mathrm{a}$ & $76.57 \mathrm{a}$ & $113.13 \mathrm{a}$ & $155.14 \mathrm{a}$ & $136.15 a$ & $183.69 \mathrm{a}$ & $14.18 \mathrm{a}$ & $19.86 \mathrm{a}$ \\
\hline & S3 & $855.49 a$ & $632.78 \mathrm{a}$ & $1316.10 \mathrm{a}$ & $1579.92 \mathrm{a}$ & $69.92 \mathrm{a}$ & $58.95 \mathrm{abc}$ & $166.29 \mathrm{a}$ & $172.81 \mathrm{a}$ & $155.12 \mathrm{a}$ & $158.05 \mathrm{a}$ & $16.88 \mathrm{a}$ & $16.55 \mathrm{a}$ \\
\hline \multirow{3}{*}{ N3 } & S1 & $644.85 \mathrm{a}$ & $632.78 \mathrm{a}$ & $1194.44 \mathrm{a}$ & $1187.58 \mathrm{a}$ & $57.66 \mathrm{a}$ & $57.14 \mathrm{bc}$ & $157.24 \mathrm{a}$ & $133.04 \mathrm{a}$ & $183.92 \mathrm{a}$ & $182.04 \mathrm{a}$ & $21.49 \mathrm{a}$ & $19.02 \mathrm{a}$ \\
\hline & $\mathrm{S} 2$ & $623.27 \mathrm{a}$ & $672.64 \mathrm{a}$ & $1073.70 \mathrm{a}$ & $1293.75 \mathrm{a}$ & $54.90 \mathrm{a}$ & $63.82 \mathrm{ab}$ & $130.49 a$ & $153.80 \mathrm{a}$ & $139.50 \mathrm{a}$ & $168.55 \mathrm{a}$ & $15.90 \mathrm{a}$ & $18.65 \mathrm{a}$ \\
\hline & $\mathrm{S3}$ & $684.73 \mathrm{a}$ & $854.17 \mathrm{a}$ & $1188.13 \mathrm{a}$ & $1600.28 \mathrm{a}$ & $61.78 \mathrm{a}$ & $77.82 \mathrm{a}$ & $162.14 \mathrm{a}$ & $177.36 \mathrm{a}$ & $133.78 \mathrm{a}$ & $175.32 \mathrm{a}$ & $15.99 \mathrm{a}$ & $18.23 \mathrm{a}$ \\
\hline \multicolumn{2}{|c|}{ F-test } & $\mathrm{NS}$ & $\mathrm{NS}$ & NS & $\mathrm{NS}$ & NS & * & $\mathrm{NS}$ & NS & $\mathrm{NS}$ & NS & NS & NS \\
\hline \multicolumn{2}{|c|}{$\mathrm{SE} \pm$} & 132.04 & 63.97 & 162.70 & 153.99 & 8.42 & 5.91 & 13.13 & 14.99 & 22.83 & 1729 & 1.68 & 1.66 \\
\hline
\end{tabular}

NS: Not significant $\quad$ *: Significant (5\%) $\quad$ **: Highly Significant (1\%)

Means within each column followed by the same letters are not significantly different at (5\%) level according to DMRT.

Nitrogen fertilization: N0 (Control) N1 (40 kg, N/ha) N2 (80 kg, N/ha) N3 (120 kg, N/ha)

Spacing: $\mathrm{S} 1(15 \mathrm{~cm}) \quad \mathrm{S} 2(20 \mathrm{~cm}) \quad \mathrm{S} 3(25 \mathrm{~cm})$ 
Agric. Biol. J. N. Am., 2011, 2(5): 791-798

Table 3: Effects of nitrogen $x$ cultivar interactions on yield and yield components of fodder beet for 2007-08 and 2008-09

\begin{tabular}{|c|c|c|c|c|c|c|c|c|c|c|c|c|c|}
\hline \multicolumn{2}{|c|}{ Treatments } & \multicolumn{2}{|c|}{$\begin{array}{l}\text { Shoot fresh weight } \\
\text { (g) }\end{array}$} & \multicolumn{2}{|c|}{$\begin{array}{l}\text { Root fresh weight } \\
\text { (g) }\end{array}$} & \multicolumn{2}{|c|}{$\begin{array}{l}\text { Shoot dry weight } \\
\text { (g) }\end{array}$} & \multicolumn{2}{|c|}{$\begin{array}{l}\text { Root dry weight } \\
\text { (g) }\end{array}$} & \multicolumn{2}{|c|}{$\begin{array}{l}\text { Green fodder yield } \\
\text { (ton/ha }\end{array}$} & \multicolumn{2}{|c|}{$\begin{array}{l}\text { Dry fodder yield } \\
\text { (ton/h }\end{array}$} \\
\hline & & $2007 / 08$ & $2008 / 09$ & $2007 / 08$ & $2008 / 09$ & $2007 / 08$ & $2008 / 09$ & $2007 / 08$ & $2008 / 09$ & $2007 / 08$ & $2008 / 09$ & $2007 / 08$ & $2008 / 09$ \\
\hline \multirow{3}{*}{ NO } & CV1 & $542.25 \mathrm{a}$ & $584.44 \mathrm{a}$ & $778.11 \mathrm{a}$ & $1114.44 \mathrm{a}$ & $50.39 \mathrm{a}$ & $58.11 \mathrm{a}$ & $115.82 \mathrm{a}$ & $143.07 \mathrm{ab}$ & $111.20 \mathrm{a}$ & $142.30 \mathrm{a}$ & $115.82 \mathrm{a}$ & $16.85 \mathrm{a}$ \\
\hline & CV2 & $396.94 \mathrm{a}$ & $372.64 \mathrm{a}$ & $793.44 \mathrm{a}$ & $968.90 \mathrm{a}$ & $42.57 \mathrm{a}$ & $35.90 \mathrm{a}$ & $107.45 \mathrm{a}$ & $124.73 b$ & $99.37 \mathrm{a}$ & $116.14 \mathrm{a}$ & $107.45 \mathrm{a}$ & $13.73 \mathrm{a}$ \\
\hline & CV3 & $490.75 \mathrm{a}$ & $510.00 \mathrm{a}$ & $829.13 \mathrm{a}$ & $1010.70 \mathrm{a}$ & $46.36 \mathrm{a}$ & $50.49 a$ & $119.40 \mathrm{a}$ & $118.51 b$ & $109.84 \mathrm{a}$ & $130.66 \mathrm{a}$ & $119.40 \mathrm{a}$ & $14.48 \mathrm{a}$ \\
\hline \multirow{3}{*}{ N1 } & CV1 & $588.00 \mathrm{a}$ & $513.61 \mathrm{a}$ & $1046.94 \mathrm{a}$ & $1011.81 \mathrm{a}$ & $55.08 \mathrm{a}$ & $48.99 \mathrm{a}$ & 141.84 & $140.17 \mathrm{ab}$ & $138.37 \mathrm{a}$ & $129.69 \mathrm{a}$ & $141.84 \mathrm{a}$ & $16.14 \mathrm{a}$ \\
\hline & $\mathrm{CV} 2$ & $529.40 \mathrm{a}$ & $440.14 \mathrm{a}$ & $979.14 \mathrm{a}$ & $1177.36 \mathrm{a}$ & $47.54 \mathrm{a}$ & $43.40 \mathrm{a}$ & $125.57 \mathrm{a}$ & 164.19 a & $129.36 \mathrm{a}$ & $141.28 \mathrm{a}$ & $125.57 \mathrm{a}$ & $17.72 \mathrm{a}$ \\
\hline & $\mathrm{CV} 3$ & $558.44 \mathrm{a}$ & $575.00 \mathrm{a}$ & $870.01 \mathrm{a}$ & $1205.42 \mathrm{a}$ & $49.95 \mathrm{a}$ & $47.61 \mathrm{a}$ & $118.53 \mathrm{a}$ & 160.32 a & $119.62 \mathrm{a}$ & $152.98 \mathrm{a}$ & $118.53 \mathrm{a}$ & $17.80 \mathrm{a}$ \\
\hline \multirow{3}{*}{$\mathrm{N} 2$} & CV1 & $805.99 \mathrm{a}$ & $771.11 \mathrm{a}$ & $1055.64 \mathrm{a}$ & $1270.14 \mathrm{a}$ & $64.18 \mathrm{a}$ & $71.32 \mathrm{a}$ & $131.68 \mathrm{a}$ & $145.93 \mathrm{ab}$ & $156.36 \mathrm{a}$ & $175.10 \mathrm{a}$ & $131.68 \mathrm{a}$ & $18.58 \mathrm{~s}$ \\
\hline & CV2 & $535.88 \mathrm{a}$ & $545.27 \mathrm{a}$ & $987.97 \mathrm{a}$ & $1500.95 \mathrm{a}$ & $48.73 \mathrm{a}$ & $52.04 \mathrm{a}$ & $132.30 \mathrm{a}$ & 168.75 a & $127.84 \mathrm{a}$ & $174.93 \mathrm{a}$ & $132.30 \mathrm{a}$ & $19.03 \mathrm{a}$ \\
\hline & CV3 & $755.42 \mathrm{a}$ & $788.50 \mathrm{a}$ & $1048.88 \mathrm{a}$ & $1488.08 \mathrm{a}$ & $62.68 \mathrm{a}$ & $72.53 \mathrm{a}$ & $131.48 \mathrm{a}$ & $170.74 \mathrm{a}$ & $150.01 \mathrm{a}$ & $192.53 \mathrm{a}$ & $131.48 \mathrm{a}$ & $20.60 a$ \\
\hline \multirow{3}{*}{ N3 } & CV1 & $640.56 \mathrm{a}$ & $745.28 \mathrm{a}$ & $1024.17 \mathrm{a}$ & $1403.20 \mathrm{a}$ & $58.74 \mathrm{a}$ & $68.04 \mathrm{a}$ & $136.53 \mathrm{a}$ & $159.94 \mathrm{a}$ & $135.24 \mathrm{a}$ & $181.05 \mathrm{a}$ & $136.53 \mathrm{a}$ & $19.25 \mathrm{a}$ \\
\hline & $\mathrm{CV} 2$ & $519.59 \mathrm{a}$ & $650.97 \mathrm{a}$ & $1236.28 \mathrm{a}$ & $1360.36 \mathrm{a}$ & $47.28 \mathrm{a}$ & $58.70 \mathrm{a}$ & $158.37 \mathrm{a}$ & $141.80 \mathrm{ab}$ & $152.80 \mathrm{a}$ & $170.77 \mathrm{a}$ & $158.37 \mathrm{a}$ & $17.10 \mathrm{a}$ \\
\hline & CV3 & $792.72 \mathrm{a}$ & $763.33 \mathrm{a}$ & $1195.83 \mathrm{a}$ & $1318.06 \mathrm{a}$ & $68.31 \mathrm{a}$ & $72.05 \mathrm{a}$ & $154.98 \mathrm{a}$ & $162.46 \mathrm{a}$ & $169.16 \mathrm{a}$ & $174.09 \mathrm{a}$ & $154.98 \mathrm{a}$ & $19.55 a$ \\
\hline \multicolumn{2}{|c|}{$\begin{array}{l}\text { F-test } \\
\mathrm{SE}+\end{array}$} & $\begin{array}{c}\text { NS } \\
63.94\end{array}$ & $\begin{array}{c}\text { NS } \\
54.38\end{array}$ & $\begin{array}{c}\text { NS } \\
99.92\end{array}$ & $\begin{array}{c}\text { NS } \\
102.97\end{array}$ & $\begin{array}{l}\mathrm{NS} \\
4.81\end{array}$ & $\begin{array}{c}N S \\
4.93\end{array}$ & $\begin{array}{c}\text { NS } \\
10.03\end{array}$ & * & $\begin{array}{c}\text { NS } \\
1283\end{array}$ & $\begin{array}{c}\text { NS } \\
1217\end{array}$ & $\begin{array}{c}\text { NS } \\
10.03\end{array}$ & $\begin{array}{c}\text { NS } \\
1.14\end{array}$ \\
\hline
\end{tabular}

NS: Not significant $\quad *$ : Significant $(5 \%) \quad$ **: Highly Significant $(1 \%)$

Means within each column followed by the same letters are not significantly different at (5\%) level according to DMRT.

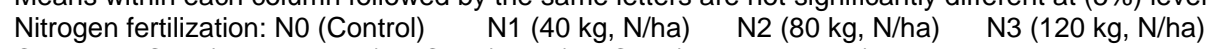

Cultivars: CV1 (Voroshenger) CV2 (Anisa) CV3 (Polyproductiva)

Table 4: Effects of spacing x cultivar interactions on yield and yield components of fodder beet for 2007-08 and 2008-09

\begin{tabular}{|c|c|c|c|c|c|c|c|c|c|c|c|c|c|}
\hline \multirow{2}{*}{\multicolumn{2}{|c|}{ Treatments }} & \multicolumn{2}{|c|}{$\begin{array}{l}\text { Shoot fresh weight } \\
(\mathrm{g})\end{array}$} & \multicolumn{2}{|c|}{$\begin{array}{l}\text { Root fresh weight } \\
\text { (g) }\end{array}$} & \multicolumn{2}{|c|}{$\begin{array}{l}\text { Shoot dry weight } \\
\text { (g) }\end{array}$} & \multicolumn{2}{|c|}{$\begin{array}{l}\text { Root dry weight } \\
\text { (g) }\end{array}$} & \multicolumn{2}{|c|}{$\begin{array}{l}\text { Green fodder yield } \\
\text { (ton/ha) }\end{array}$} & \multicolumn{2}{|c|}{$\begin{array}{l}\text { Dry fodder yield } \\
\text { (ton/ha) }\end{array}$} \\
\hline & & $2007 / 08$ & $2008 / 09$ & $2007 / 08$ & $2008 / 09$ & $2007 / 08$ & $2008 / 09$ & $2007 / 08$ & $2008 / 09$ & $2007 / 08$ & $2008 / 09$ & $2007 / 08$ & $2008 / 09$ \\
\hline \multirow{3}{*}{ S1 } & CV1 & $520.69 a$ & $613.85 \mathrm{a}$ & $841.54 \mathrm{a}$ & $1044.17 \mathrm{a}$ & $47.34 \mathrm{a}$ & $58.60 \mathrm{a}$ & $120.91 \mathrm{a}$ & $136.22 \mathrm{a}$ & $136.22 \mathrm{a}$ & $165.80 \mathrm{a}$ & $16.83 \mathrm{a}$ & $18.85 \mathrm{a}$ \\
\hline & CV2 & $441.96 \mathrm{a}$ & $506.39 a$ & $954.54 \mathrm{a}$ & $1305.76 \mathrm{a}$ & $44.68 \mathrm{a}$ & $47.15 \mathrm{a}$ & $126.86 \mathrm{a}$ & $139.66 \mathrm{a}$ & $139.66 \mathrm{a}$ & $181.22 \mathrm{a}$ & $17.15 \mathrm{a}$ & $20.35 a$ \\
\hline & CV3 & $570.88 \mathrm{a}$ & $629.58 \mathrm{a}$ & $826.29 \mathrm{a}$ & $1143.12 \mathrm{a}$ & $54.05 \mathrm{a}$ & $56.25 \mathrm{a}$ & $117.49 \mathrm{a}$ & $139.71 \mathrm{a}$ & $139.71 \mathrm{a}$ & $177.27 \mathrm{a}$ & $17.33 \mathrm{a}$ & $19.04 \mathrm{~d}$ \\
\hline \multirow{3}{*}{ S2 } & CV1 & $687.81 \mathrm{a}$ & $643.33 \mathrm{a}$ & $1005.91 \mathrm{a}$ & $1215.00 \mathrm{a}$ & $58.29 \mathrm{a}$ & $61.72 \mathrm{a}$ & $132.16 \mathrm{a}$ & $140.72 \mathrm{a}$ & $140.72 \mathrm{a}$ & $159.29 \mathrm{a}$ & $16.33 \mathrm{a}$ & $17.82 \mathrm{a}$ \\
\hline & CV2 & $473.13 \mathrm{a}$ & $462.25 \mathrm{a}$ & $989.40 \mathrm{a}$ & $1202.71 \mathrm{a}$ & $44.70 \mathrm{a}$ & $44.81 \mathrm{a}$ & $126.01 \mathrm{a}$ & $125.36 \mathrm{a}$ & $125.36 \mathrm{a}$ & $142.71 \mathrm{a}$ & $14.63 \mathrm{a}$ & $16.46 \mathrm{a}$ \\
\hline & $\mathrm{CV} 3$ & $580.15 \mathrm{a}$ & $670.75 \mathrm{a}$ & $898.83 \mathrm{a}$ & $1200.42 \mathrm{a}$ & $50.36 \mathrm{a}$ & $65.58 \mathrm{a}$ & $117.37 \mathrm{a}$ & $126.77 \mathrm{a}$ & $126.77 \mathrm{a}$ & $160.39 \mathrm{a}$ & $14.38 \mathrm{a}$ & $18.54 \mathrm{a}$ \\
\hline \multirow{3}{*}{ S3 } & CV1 & $724.09 \mathrm{a}$ & $703.65 \mathrm{a}$ & $1081.19 \mathrm{a}$ & $1340.52 \mathrm{a}$ & $65.67 \mathrm{a}$ & $64.52 \mathrm{a}$ & $141.33 \mathrm{a}$ & $128.94 \mathrm{a}$ & $128.94 \mathrm{a}$ & $146.01 \mathrm{a}$ & $14.79 \mathrm{a}$ & $16.45 \mathrm{a}$ \\
\hline & CV2 & $571.27 \mathrm{a}$ & $538.13 \mathrm{a}$ & $1066.80 \mathrm{a}$ & $1247.22 \mathrm{a}$ & $50.21 \mathrm{a}$ & $50.58 a$ & $139.89 a$ & $117.01 \mathrm{a}$ & $117.01 \mathrm{a}$ & $128.42 \mathrm{a}$ & $13.58 \mathrm{a}$ & $13.87 \mathrm{a}$ \\
\hline & CV3 & $796.97 \mathrm{a}$ & $677.29 \mathrm{a}$ & $1232.76 \mathrm{a}$ & $1423.15 \mathrm{a}$ & $66.06 \mathrm{a}$ & $60.17 \mathrm{a}$ & $158.43 \mathrm{a}$ & $144.98 \mathrm{a}$ & $144.98 \mathrm{a}$ & $150.03 \mathrm{a}$ & $16.04 \mathrm{a}$ & $16.75 \mathrm{a}$ \\
\hline \multicolumn{2}{|c|}{$\begin{array}{l}\text { F-test } \\
\text { SE } \pm\end{array}$} & $\begin{array}{c}N S \\
55.37\end{array}$ & $\begin{array}{c}\text { NS } \\
47.09\end{array}$ & $\begin{array}{c}\text { NS } \\
86.53\end{array}$ & $\begin{array}{c}\text { NS } \\
89.17\end{array}$ & $\begin{array}{c}\mathrm{NS} \\
4.17\end{array}$ & $\begin{array}{c}\text { NS } \\
4.27\end{array}$ & $\begin{array}{c}N S \\
8.68\end{array}$ & $\begin{array}{l}* \\
8.96\end{array}$ & $\begin{array}{c}\text { NS } \\
11.11\end{array}$ & $\begin{array}{c}\text { NS } \\
12.17\end{array}$ & $\begin{array}{c}\mathrm{NS} \\
0.93\end{array}$ & $\begin{array}{l}\text { NS } \\
0.99\end{array}$ \\
\hline
\end{tabular}

NS: Not significant $\quad *$ : Significant $(5 \%) \quad$ **: Highly Significant $(1 \%)$

Means within each column followed by the same letters are not significantly different at (5\%) level according to DMRT.

Cultivars: CV1 (Voroshenger) CV2 (Anisa) CV3 (Polyproductiva) 
planting spacing was found to be inversely related to green fodder yield. Spacing of $15 \mathrm{~cm}\left(\mathrm{~S}_{1}\right)$ had significantly higher yield than $25 \mathrm{~cm}\left(\mathrm{~S}_{3}\right)$ which was not significantly different from $20 \mathrm{~cm}\left(\mathrm{~S}_{2}\right)$ (Table 1). Similar observation was made by Augustinussen (1974). Also, Kamel et al. (1990) reported that total fresh yield of fodder beet was reduced by increasing plant spacing. In closer spacing there was greater number of plants, compared to wider spacing, which resulted in greater yield. The lack of cultivar differences in green fodder yield as shown in table (I) was consistent with results reported by Nadaf et al. (1998a).

Dry fodder yield: Dry fodder yield increased as a result of increasing nitrogen fertilizer levels (Table I). Increasing nitrogen level up to $120 \mathrm{~kg} / \mathrm{ha}\left(\mathrm{N}_{3}\right)$ significantly increased dry yield over the control by $23.8 \%$ in the first season (Table 1). This may be due to the effect of nitrogen in enhancing photosynthesis and dry matter production. Similar results were obtained by Ouda and Sohier (2005), Saif (2000) and Ouda (2002).Dry fodder yield was increased significantly when beet was sown at denser spacing $(15 \mathrm{~cm})$ compared to wider ones $(20$ and $25 \mathrm{~cm})$ (Table 1).This agrees with Hills and Worker (1974) and Kamel et al. (1990). The cultivars of Voroshenger, Anisa and Polyproductiva were comparable in dry fodder yield (Table 1). Analogous result was reported by Al-bayrak and Camas (2006) who reported that Ecdogelb and Ecdorot fodder beet cultivars were not significantly different in fresh fodder yield.

\section{CONCLUSIONS:}

* $\quad$ Nitrogen fertilization improved yield of fodder beet, and had significant positive effects on root fresh weight, shoot dry weight, and root dry weight, green and dry fodder yields. Mostly, the highest yield and yield components were associated with $80 \mathrm{~kg}$ $\mathrm{N} / \mathrm{ha}$. So, it can be recommended to be the optimum dose under Khartoum conditions.

There was a trend for yield components to increase at wider plant spacing of $25 \mathrm{~cm}$. However, the highest $(p<0.05)$ green and dry fodder yields were obtained under closer spacing of $15 \mathrm{~cm}$, which may be due to higher number of plants. So, it is recommended to plant beet at $15 \mathrm{~cm}$ apart at Shambat area.
* Both Voroshenger and Polyproductiva cultivars were superior over Anisa in yield and yield components. But, there was no significant difference between the three tested cultivars in yields. Henceforth, the three cultivars are recommended to be planted.

* $\quad$ The high yields (approximately 60 and 7 ton per feddan of green and dry fodder yield, respectively) shown by all cultivars tested may be attributed to the favorable growing conditions for the crop and are indication of the adaptability of fodder beet, as far as yield is concerned, to the environmental conditions at the experimental site.

* $\quad$ Most interactions between nitrogen, spacing and cultivars were not significant for yield and yield components.

* The present results are, indicative of the potential success of fodder beet as a winter fodder crop in Sudan. Since climatic and agronomic factors can influence the performance and success of beet culture, hence, further trials are required to identify optimum agronomic practices especially sowing time, harvesting date, soil type, land preparation, fertilization and spacing .

\section{ACKNOWLEDEMENT}

We acknowledge with great gratitude the Scientific Research Council-Sudan University of Science and Technology for financing this research. Fresh and dry weight of root, green and dry fodder yields were not significantly affected by cultivars. Shoot dry weight was significantly greater under higher nitrogen levels and wider spacing $\left(\mathrm{N}_{2} \mathrm{~S}_{2}\right.$ and $\left.\mathrm{N}_{3} \mathrm{~S}_{3}\right)$ in the second season. Nitrogen and cultivars interaction resulted in significant $(p<0.05)$ increase in root dry weight attained by Voroshenger and Polyproductiva cultivars under $80 \mathrm{~kg} / \mathrm{N} / \mathrm{ha}\left(\mathrm{N}_{2} \mathrm{~V}_{1}\right.$ and $\left.\mathrm{N}_{2} \mathrm{~V}_{3}\right)$ in the first and second season, respectively. Polyproductiva sown at $25 \mathrm{~cm}$ apart $\left(\mathrm{S}_{3} \mathrm{~V}_{3}\right)$ significantly $(\mathrm{p}<0.05)$ attained the greatest root dry weight in the second season.

\section{REFERENCES:}

Abdalla, R.S. (1999). The response of two Sugar beet (Beta vulgaris) cultivars to sowing date and harvest time under Central Sudan environment. M.Sc. Thesis, Faculty of Agriculture, University of Khartoum, Sudan. 
Albayrak, S. and Camas, N. (2006). Yield components of Fodder beet (Beta vulgaris var. crassa mansf.) under the middle Black Sea region conditions. Tarim Bilimleri Dergisi, 12 (1): 65-69.

Anonymous (2006). http:// www. Seed 2 grow. Co. UK/acatalogy/Fodder-Beet- seed.himl (2006).

Augustinussen, E. (1974). Yield and quality of Fodder beet as affected by high density of stand. Tidsskrift for planteavl, 78 (2): 191-202.

Basha, H.A. (1998). Effect of hill spacing and nitrogen split application on Fodder beet in newly cultivated sandy soil. Zagazig Journal of Agricultural Research, 25 (1): 59-71.

Basha, H.A. (1999). Response of two Sugar beet cultivars to level and method of nitrogen application in sandy soil. Zagazig Journal of Agricultural Research, 26 (1): 11-26.

Blumenthal, Jurg, M., Baltensperger, David., D., Cassman, Kenneth, G., Mason, Stephen, C. and Pavlista, Alexander, Alexander, D. (2008).Importance and Effect of Nitrogen on Crop Quality and Halth. In: Nitrogen in the Environment: Source Problems and Management. $2^{\text {nd }}$ edition. Elsevier Inc. Amsterdam, pp. 51.

DAF (Department of Agriculture and Food) (1988). Root, fodder crop, pulse and oilseed varieties. Irish recommendd list. Government Stationary Office, Dublin, 17p. [C.F. Albayrak, S. and Camas, N. (2006). Yield components of Fodder beet (Beta vulgaris var. crassa mansf.) under the middle Black Sea region conditions. Tarim Bilimleri Dergisi, 12 (1): 65-69].

El-Shafai, A.M.A. (2000). Effect of nitrogen and potassium fertilization on yield and quality of Sugar beet in Sohag. Egyptian Journal of Agricultural Research, 78 (2): 759767.

Gomez, K.A. and Gomez, A.A. (1984). Statistical procedures for agricultural research. $2^{\text {nd }}$ edition, john Willy and sons Inc. New York. 680p.

Hassanin, M.A.and Ramadan, B.H. (1999). Influence of plant densities and distribution on performance of some Sugar beet varieties (Beta vulgaris L.). Bull. Faculty of Agriculture, Cairo University (11): 315-322.

Hills, F.G. and Worker, G.F. (1974). Spacing Sugar beet for maximum production. California Agric., 28: 4-15. [C.F. Mousa, M.E. and Sarhan, G.M. (1996). Effect of varietal variation on growth and yield of Fodder beet (Beta vulgaris L.) in middle Egypt. Zagazig Journal of Agricultural Research, 23 (3): 333-344].
HTTP://www.biologie.uni-hamburg.de/bonline/schaugaren/varalba/fodder-beet (2006).

Ibrahim, M.F.M. (1998). The effect of some fertilization elements on the yield and quality of Sugar beet. Ph.D. Thesis, Faculty of Agriculture, Moshtohor, Zagazig University, Egypt.

Ibrahim, M.M., El-Aref, KH.A.O. and Abo El-Hamd, A.S. (2005). Effect of nitrogen and phosphorus fertilization on yield and quality of two Sugar beet varieties under Assuit Governorate condition., Zagazig Journal of Agricultural Research, 32 (4): 1087-1103.

Ibrahim, Y.M. (2005). Ranges and forage (In Arabic). Dar Azza for Publication, Khartoum, Sudan, 300p.

Ibrahim, Z.O. (2008). Effect of feeding Rhodes grass (Chloris gayana) on milk production and its composition of dairy cows. M.Sc. Thesis, Faculty of Agricultural Studies, Sudan University of Science and Technology, Sudan.

Ismail, A.M.A. (2002). Evaluation of some Sugar beet varieties under different nitrogen levels in El-Fayium. Egyptian Journal of Applied Sciences, 17 (2): 75-85.

Kamel, M.S., Shaban, SH.A. Abou Deya, I.B. and Nassar, Z.M. (1990). Nitrogen fertilization and hill-spacing in Fodder beet. Yield and Chemical composition. Proc. $4^{\text {th }}$ Conf. Agron., Cairo, 15-16 Sept. Vol. II: 269-284.

Kandil, A.A., Badawi, M.A., El-Moursy, S.A. and Abdou, U.M.A. (2002). Effect of planting dates, nitrogen levels and bio-fertilization treatments: yield, yield components and quality of Sugar beet (Beta vulgaris L.). Journal of Agricultural science, Mansoura University, 27 (11): 7257-7266.

Khair, M.A.M. (1999).Principles of forage production (in Arabic). $1^{\text {st }}$ edition. Publisher: Training and Publication Administration, Agricultural Research Corporation, Sudan, 219p.

Kiely, P.O., Moloney, A.P. and Meagher, J. (1991). Ensiling and feeding whole-crop Fodder beet. Landbauforchung-voelkerode Sonderheft, 123: 269272.

Kipps, M.S. (1970). Production of field crops (A textbook of Agronomy). Sixth edition. McGraw-Hill, Inc. New York.790 p.

Mahmoud, E.A., Khalil, N.A. and Besheet, S.Y. (1990). Effect of nitrogen fertilization and plant density on Sugar beet, root weight, root, top and sugar yields and sugar quality. Proc. $4^{\text {th }}$ Conf. Agron., Cairo, 15-16 Sept., Vol. II: 433-446. 
Martin, J.H., Leonard, W.H. and Stamp, D.L. (1976). Principles of field crop production. Third edition. MacMillan Publishing Co., Inc., New York.1118p.

Mstat, V.4. (1986): A microcomputer program for the design management and analysis of agronomic research experiments. Michigan State University, USA.

Mustafa, M. El. (2007). Effect of nitrogen and phosphorus fertilization on the performance of three Sugar beet (Beta vulgaris L.) cultivars. M.Sc. Thesis, Faculty of Agriculture, University of Khartoum, Sudan.

Nadaf, S.K., Ibrhaim, Y.M., Akhtar, M., El Hag, M.G. and Al-Lawati, A.H. (1998a). Performance of Fodder beet in Oman. Annals of Arid Zone, 37 (4): 377-382.

Nadaf, S.K., Al-Khamisi, S., El Hag, M.G., Al-Lawati, A.H. and Ibrahim, Y.M. (1998b). Regional workshop on management of soils and crops. Arab Organization for Agricultural Development (AOAD), Muscat, Oman.

Nafei, A.I. (2004). Effect of nitrogen and boron fertilization levels on yield and quality of Sugar beet grown in Upper Egypt. Egyptian Journal of Applied Sciences, 19(2): 48-57.

Nemeat Alla, E.A.E., Mohammed, A.A.E. and Zalat, S.S. (2002). Effect of soil and foliar application of nitrogen fertilization on Sugar beet. Journal of Agricultural Sciences, Mansoura University, 27 (3): 1343-1351.
Ouda, Soheir, M.M. (2002). Effect of nitrogen and sulpher fertilizers levels on Sugar beet in newly cultivated sandy soil. Zagazig Journal of Agricultural Research, 29 (1): 23-50.

Ouda, Soheir, M.M. (2005). Yield and quality of Sugar beet as affected by planting density and nitrogen fertilizer levels in the newly reclaimed soil. Zagazig Journal of Agricultural Research, 32 (3): 701-715.

Saif, Lailla, M. (2000). Stepwise regression and path coefficient analysis for some Sugar beet characters under levels of boron and nitrogen fertilization. Proc. $9^{\text {th }}$ Conf. Agron., Minufiya University, 1-2 Sept. 2000, 569581.[C.F. Ouda, Soheir, M.M. (2005). Yield and quality of Sugar beet as affected by planting density and nitrogen fertilizer levels in the newly reclaimed soil. Zagazig Journal of Agricultural Research, 32 (3): 701715.

Shalaby, A.S., Rammah, A.M., Abdul-Aziz, G.M. and Beshay, M.G. (1989). Fodder beet, a new forage in Egypt. 1. Productivity and the chemical analysis of some Fodder beet (Beta vulgaris L.) cultivars sown at different locations in Egypt. In proceedings of the third Egyptian British Conference on Animals, fish and poultry production. Alexandria, Egypt, 13: 133143.[C.F. Nadaf, S.K., Ibrhaim, Y.M., Akhtar, M., El Hag, M.G. and Al-Lawati, A.H. (1998a). Performance of Fodder beet in Oman. Annals of Arid Zone, 37 (4): 377382]. 